\title{
Modulation of the Nur77-Bcl-2 apoptotic pathway by p38 $\alpha$ MAPK
}

\author{
Jie Liu' ${ }^{1, *}$, Guang-Hui Wang ${ }^{1, *}$, Ying-Hui Duan², Yi Dai ${ }^{2}$, Yuzhou Bao ${ }^{1}$, Mengjie Hu${ }^{1}$, \\ Yu-Qi Zhou ${ }^{1}$, Mingyu Li ${ }^{1}$, Fuquan Jiang ${ }^{1}$, Hu Zhou ${ }^{1}$, Xin-Sheng Yao ${ }^{2}$ and Xiao-Kun \\ Zhang ${ }^{1,3}$ \\ ${ }^{1}$ School of Pharmaceutical Sciences, Fujian Provincial Key Laboratory of Innovative Drug Target Research, Xiamen University, \\ Xiamen, China \\ ${ }^{2}$ Institutes of Traditional Chinese Medicine and Natural Products, Jinan University, Guangzhou, China \\ ${ }^{3}$ Sanford Burnham Prebys Medical Discovery Institute, La Jolla, California, USA \\ *These authors contributed equally to this work \\ Correspondence to: Xiao-Kun Zhang, email: xzhang@sbpdiscovery.org \\ Xin-Sheng Yao, email: tyaoxs@jnu.edu.cn \\ Keywords: CCE9, Nur77, BCl-2, p38 $\alpha$ MAPK, apoptosis \\ Received: March 01, $2017 \quad$ Accepted: June 05, $2017 \quad$ Published: July 13, 2017 \\ Copyright: Liu et al. This is an open-access article distributed under the terms of the Creative Commons Attribution License \\ 3.0 (CC BY 3.0), which permits unrestricted use, distribution, and reproduction in any medium, provided the original author and \\ source are credited.
}

\section{ABSTRACT}

Orphan nuclear receptor Nur77 promotes apoptosis by targeting mitochondria through interaction with $\mathrm{Bcl}-2$, an event that converts $\mathrm{Bcl}-2$ from a survival to killer. However, how the Nur77-Bcl-2 apoptotic pathway is regulated remains largely unknown. In this study, we examined the regulation of the Nur77-Bcl-2 pathway by CCE9, a xanthone compound. Our results demonstrated that the apoptotic effect of CCE9 depended on its induction of Nur77 expression, cytoplasmic localization, and mitochondrial targeting. The activation of the Nur77-Bcl-2 pathway by CCE9 was associated with its activation of p38 $\alpha$ MAPK. Inhibition of p38 $\alpha$ MAPK activation by knocking down or knocking out p38 $\alpha$ MAPK impaired the effect of CCE9 on inducing apoptosis and the expression and cytoplasmic localization of Nur77. In addition, CCE9 activation of $\mathrm{p} 38 \alpha$ MAPK resulted in $\mathrm{Bcl}-2$ phosphorylation and $\mathrm{Bcl}-2$ interaction with Nur77, whereas inhibition of p38 $\alpha$ MAPK activation or expression suppressed the interaction. Moreover, mutating Ser87 and Thr56 in the loop of Bcl-2, which are known to be phosphorylated by $\mathrm{p} 38 \alpha$ MAPK, impaired the ability Bcl-2 to interact with Nur77. Together, our results reveal a profound role of p38 $\alpha$ MAPK in regulating the Nur77-Bcl-2 apoptotic pathway through its modulation of Nur77 expression, Bcl-2 phosphorylation, and their interaction.

\section{INTRODUCTION}

Nur77 (also called TR3, NGFI-B, or NR4A1), an early immediate-response gene, is perhaps the most potent pro-apoptotic member of the nuclear receptor superfamily [1-4]. Besides being a nuclear transcriptional factor, Nur77 acts nongenomically in the cytoplasm by targeting mitochondria to induce apoptosis in response to certain apoptotic stimuli [5-15]. Mitochondrial targeting of Nur77 involves its interaction with Bcl-2 [7, 13, 15-18]. The interaction results in a Bcl-2 conformational change that converts Bcl-2 from an anti-apoptotic to a pro-apoptotic molecule [7, 13, 15-18]. Interestingly, the Nur77-Bcl-2 interaction is mediated by an unexpected protein-protein interaction site in the natively unstructured loop of $\mathrm{Bcl}-2$ located between $\mathrm{BH} 3$ and $\mathrm{BH} 4$ domains $[7,15,18]$, which has been shown to play a critical role in regulating $\mathrm{Bcl}-2$ function $[19,20]$. However, how the binding of Nur77 to the loop of Bcl-2 is regulated remains undefined.

Previous reports indicate that phosphorylation of $\mathrm{Bcl}-2$ is an important mechanism regulating the antiapoptotic effect of Bcl-2 [19, 20]. Phosphorylation of Bcl2 is mainly mediated by multiple phosphorylation sites in its loop region $[19,20]$. Among kinases involved in 
Bcl-2 loop phosphorylation, p38 $\alpha$ MAPK that regulates a variety of cellular responses to environmental stress, proinflammatory cytokines, lipopolysaccharide (LPS) and other signals $[21,22]$ has been shown to phosphorylate Ser87 and Thr56 located within the loop of Bcl-2 [23, 24]. Phosphorylation of Bcl-2 abrogates its anti-apoptotic function in response to nerve growth factor (NGF) withdrawal or treatment with tumor necrosis factor (TNF), paclitaxel, arsenic trioxide, and NO [24, 25]. Under these conditions, Bcl-2 phosphorylation by p38 $\alpha$ MAPK leads to pro-apoptotic events [24]. Interestingly, apoptosis provoked by several stimuli that activate p38 $\alpha$ MAPK is closely linked to Bcl-2-expressing populations [23, 24], demonstrating the death potential of the Bcl-2 protein upon $\mathrm{p} 38 \alpha$ MAPK phosphorylation.

Xanthones, a class of three-membered heterocyclic ring compounds mainly found as secondary metabolites in higher plants and microorganisms, exert diverse biological activities, including anti-hypertensive, anti-oxidative, antithrombotic, and anti-cancer activity, depending on their structure features [26, 27]. A large number of naturally occurring and synthetic xanthones, such as psorospermin, dimethylxanthesone-4-acetic acid (DMXAA) and $\alpha$-mangostin, exert potent anti-cancer activities in a variety of cancer cells, including breast cancer, colorectal cancer, hepatoma, leukemia, and small cell lung cancer [26-29]. We reported here that 1,3,7-trihydroxy-2,4diprenylxanthone (named CCE9) isolated from the Chinese medicinal plant, Cratoxylum formosum ssp. Pruniflorum [30] is a positive regulator of the Nur77-Bcl2-dependent apoptotic pathway. Our results demonstrated that CCE9 could induce both Nur77 expression and Bcl2 phosphorylation in a p38 $\alpha$ MAPK dependent manner, resulting in Nur77 interaction with Bcl-2 and Nur77 cytoplasmic localization. Furthermore, we showed that p38 $\alpha$ MAPK phosphorylation of Ser87 and Thr56 in the loop of Bcl-2 was essential for its interaction with Nur77. Our results therefore reveal a critical role of $\mathrm{p} 38 \alpha$ MAPK in the regulation of the Nur77-Bcl-2 apoptotic pathway.

\section{RESULTS}

\section{CCE9 induces apoptosis in a Nur77 and Bcl-2 dependent manner}

To identify new modulators of the Nur77-Bcl-2 apoptotic pathway, we screened a natural product library prepared from Chinese herbal medicines, and found that CCE9 (Figure 1A) could potently increase Nur77 expression and apoptosis. In HeLa229 cells, CCE9 induced a rapid increase of Nur77 expression with maximum induction in cells treated with CCE9 for $3 \mathrm{hr}$ and $6 \mathrm{hr}$ (Figure 1B). CCE9 also showed a dose-dependent induction of Nur77 expression. Although CCE9 induction of Nur77 could be seen at $1 \mu \mathrm{M}$ concentration, significant Nur77 induction was observed when $5 \mu \mathrm{M}$ or higher dose of CCE9 was used (Figure 1C). Levels of Nur77 protein in A549 and HepG2 cells were also induced by CCE9 in a time- (Figure 1D) and dose-dependent (Figure 1E) manner. We also determined whether CCE9 could induce Nur77 mRNA expression. HeLa229 cells treated with vehicle or with CCE9 at 5, 10, $20 \mu \mathrm{M}$ for $3 \mathrm{hr}$ were examined for levels of Nur77 transcript by reverse transcriptionPCR (RT-PCR). While strong induction of Nur77 mRNA expression was seen when cells were treated with phorbol12-myristate-13-acetate (TPA), no apparent induction of Nur77 mRNA level by CCE9 was found (Figure 1F). Thus, the induction of Nur77 protein by CCE9 was not due to its transcriptional regulation of Nur77 expression.

The death effect of CCE9 was examined by assessing its ability to induce PARP cleavage [5]. Our data showed that PARP was cleaved by CCE9 treatment, which correlated well with its induction of Nur77 expression (Figure 1B, 1C). CCE9 also induced both Nur77 expression and PARP cleavage in A549 cells and HepG2 cells in a time- and dose-dependent manner (Figure 1D, 1E). The apoptotic effect of CCE9 was also illustrated by its activation of caspase- 3 in HeLa229 cells revealed by immunostaining (Figure $1 \mathrm{G}$ ). In addition, DAPI staining showed that treatment of HeLa229 cells by CCE9 for 3 hr resulted in about $18.0 \%$ of cells displaying nuclear condensation and fragmentation, which was increased to $29.3 \%$ when cells were treated with CCE9 for $6 \mathrm{hr}$ (Figure 1H). Furthermore, Annexin V/PI staining showed about $19.85 \%$ HeLa229 cells undergoing extensive early apoptosis (Annexin $\mathrm{V}^{+} / \mathrm{PI}^{-}$) when they were treated with 10 $\mu \mathrm{M}$ CCE9 for $6 \mathrm{hr}$ (Figure 1I).

Our observation that CCE9-induced apoptosis was accompanied with its induction of Nur77 expression promoted us to determine whether Nur77 expression plays a role in CCE9 induction of apoptosis. Thus, Nur77 siRNA was transfected into HeLa229 cells, which almost completely suppressed the induction of Nur77 expression by CCE9 (Figure 2A). Inhibition of Nur77 expression by Nur77 siRNA transfection impaired the ability of CCE9 to induce PARP cleavage (Figure 2A). Caspase-3 activation by CCE9 was also reduced by transfection of Nur77 siRNA (Figure 2B). We also used DAPI staining to evaluate the effect of Nur77 expression. CCE9 treatment resulted in about $20.3 \%$ cells displaying apoptotic nuclear morphology such as nuclear condensation and fragmentation when cells were transfected with control siRNA, which was reduced to $11.7 \%$ when cells were transfected with Nur77 siRNA (Figure 2D). We also examined whether overexpression of Nur77 could enhance the death effect of CCE9. HeLa229 cells were transfected with GFP-Nur77 (Figure 2C) and subsequently treated with CCE9. About 55.3\% GFP-Nur77-transfected cells displayed nuclear condensation and fragmentation (Figure 2D). To further determine the role of Nur77 expression, HeLa229 cells transfected with either Nur77 siRNA or GFP-Nur77 were evaluated by Annexin V/PI staining. 

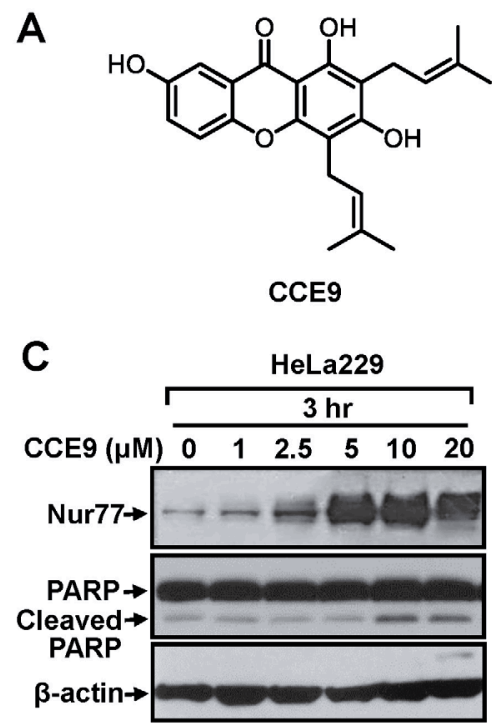

E

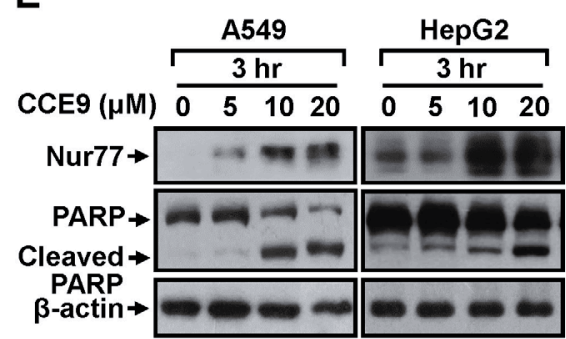

G

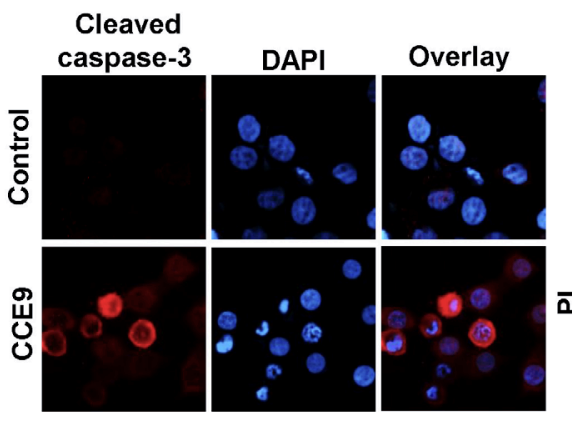

B

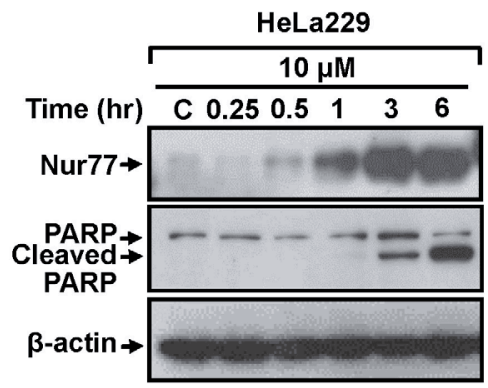

D

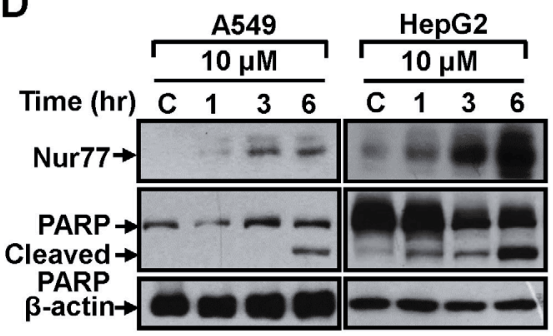

$\mathbf{F}$

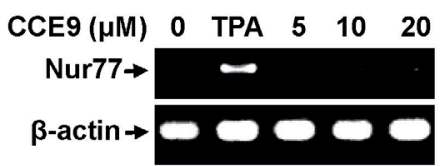

$\mathrm{H}$
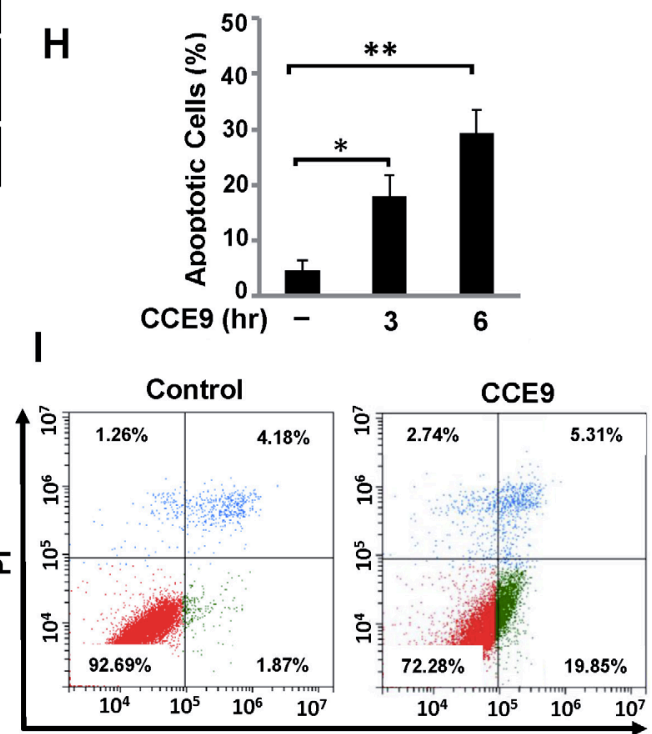

Annexin v -FITC

Figure 1: CCE9 induces Nur77 expression and apoptosis. (A) Structure of CCE9. (B) Time-course analysis of Nur77 and PARP cleavage induction by CCE9. HeLa229 cells treated with $10 \mu \mathrm{M}$ CCE9 for the indicated time were determined by Western blotting using anti-Nur77 antibody. (C) Dose dependent effect of CCE9. HeLa229 cells treated with vehicle or indicated concentration of CCE9 for 3 hr were analyzed for Nur77 expression and PARP cleavage by Western blotting. (D) Time-course analysis of Nur77 expression and apoptosis induction by CCE9 in A549 and HepG2 cells. Cells treated with $10 \mu \mathrm{M}$ CCE9 for the indicated time were analyzed for Nur77 expression and PARP cleavage by Western blotting. (E) Dose-dependent induction of Nur77 and apoptosis by CCE9 in A549 and HepG2 cells. Cells treated with vehicle or the indicated concentration of CCE9 for $3 \mathrm{hr}$ were analyzed for Nur77 expression and PARP cleavage by Western blotting. (F) RT-PCR analysis of Nur77 mRNA expression in HeLa229 cells. Cells treated with vehicle, TPA (100 ng/ml), or indicated concentration of CCE9 for $3 \mathrm{hr}$. Nur77 and $\beta$-actin mRNA products were simultaneously amplified in the same reaction system, in which $\beta$-actin expression level served as an internal control. (G) Caspase-3 activation by CCE9. HeLa229 cells were treated with $10 \mu \mathrm{M}$ CCE9 for $3 \mathrm{hr}$, immunostained with antibody recognizing the cleaved caspase-3. Nuclei were visualized by co-staining with DAPI. (H) DAPI staining. HeLa229 cells were treated with CCE9 $(10 \mu \mathrm{M})$ or vehicle for $3 \mathrm{hr}$ or $6 \mathrm{hr}$ and subjected to DAPI staining. Apoptotic cells were scored and compared between different treatments. *, $\mathrm{P}<0.01$ (VS. control); **, $\mathrm{P}<0.01$ (VS. control). (I) The apoptotic effect of CCE9. HeLa229 cells were treated with vehicle or $10 \mu \mathrm{M}$ CCE9 for $6 \mathrm{hr}$ and stained with Annexin V/PI. Apoptosis was analyzed by fluorescenceactivated cell sorting analysis. 
A

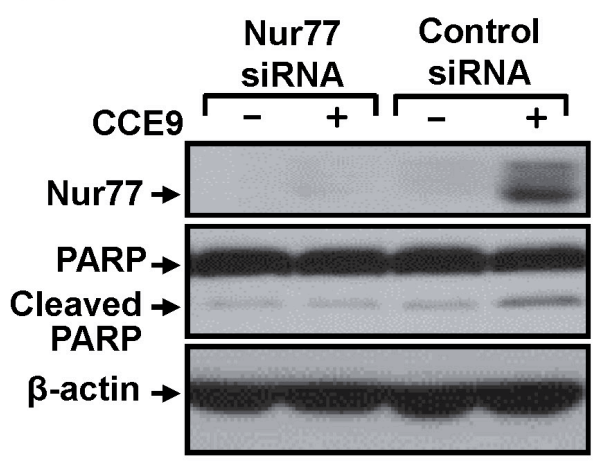

B

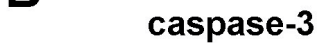

DAPI

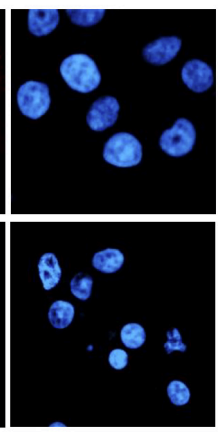

Overlay

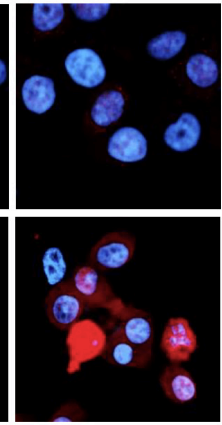

C

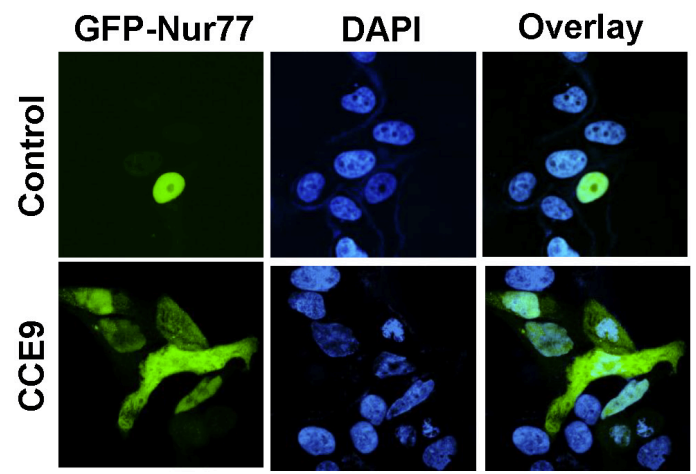

D

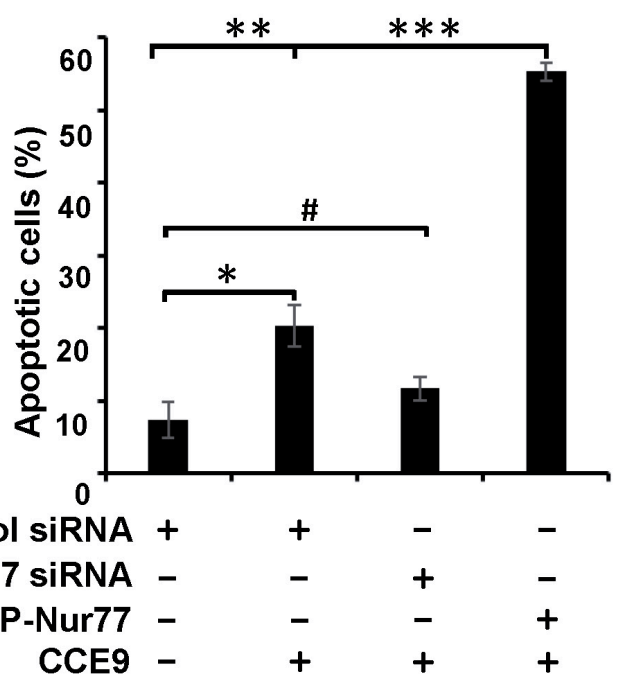

E
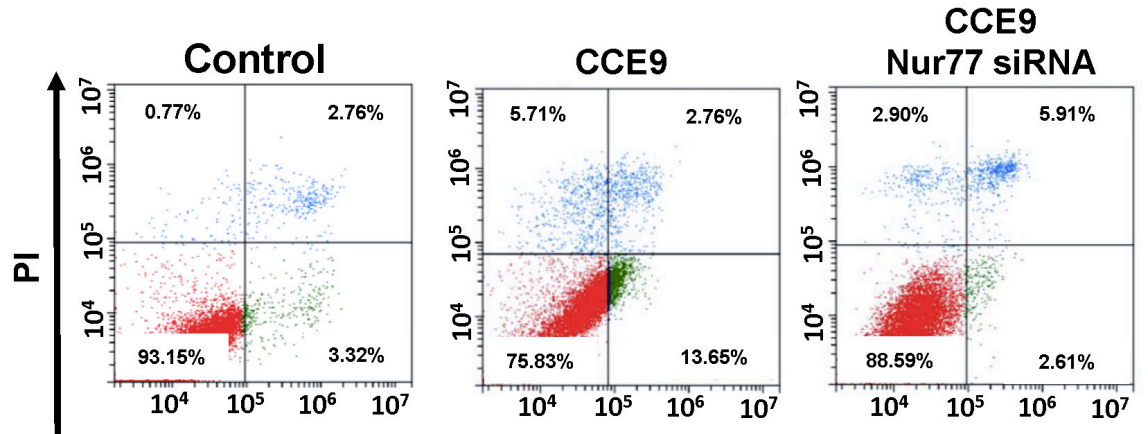

CCE9

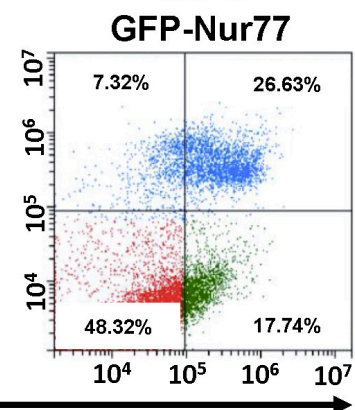

Annexin V -FITC

Figure 2: Role of Nur77 in CCE9 induced apoptosis. (A-B) Transfection of Nur77 siRNA inhibits apoptosis induction by CCE9. HeLa229 cells transfected with control or Nur77 siRNA were treated with CCE9 $(10 \mu \mathrm{M})$ for $3 \mathrm{hr}$, and analyzed for PARP cleavage by Western blotting (A), caspase-3 activation by immunostaining (B). (C) Transfection of Nur77 promotes apoptosis induction by CCE9. HeLa229 cells transfected with GFP-Nur77 expression vector were treated with $10 \mu \mathrm{M}$ of CCE9 for 3 hr. Subcellular localization of GFP-Nur77 was examined by confocal microscopy. (D) Nur77 mediates the apoptotic effect of CCE9. HeLa229 cells were transfected with Nur77 siRNA and GFP-Nur77and subjected to CCE9 treatment $(10 \mu \mathrm{M})$ or vehicle in serum-free medium for $3 \mathrm{hr}$. Apoptotic cells examined by DAPI staining were compared between different cells. *, $\mathrm{P}<0.01$ (vs. control); **, $\mathrm{P}<0.01$ (vs. control); ***, $\mathrm{P}<0.01$ (vs. control siRNA with CCE9 treatment); \#, P>0.05 (vs. control). (E) The apoptotic effect of CCE9 is impaired in Nur77 knockdown and Nur77 overexpressed cells. HeLa229 cells transfected with Nur77 siRNA and HeLa229 cells transfected with GFP-Nur77 were treated with vehicle or $10 \mu \mathrm{M}$ of CCE9 for $6 \mathrm{hr}$ and stained with Annexin V/PI. Apoptosis was analyzed by fluorescence-activated cell sorting analysis. 
Transfection of Nur77 siRNA inhibited the death effect of CCE9 on inducing early apoptosis, revealed by the reduction of the percentage of Annexin $\mathrm{V}^{+} / \mathrm{PI}^{-}$cells from $13.65 \%$ to $2.61 \%$ (Figure $2 \mathrm{E}$ ). In contrast, transfection of GFP-Nur77 enhanced the apoptotic effect of CCE9, as its induction of late apoptosis (Annexin $\mathrm{V}^{+} / \mathrm{PI}^{+}$cells) was increased from $2.76 \%$ to $26.63 \%$. Taken together, these studies demonstrated that Nur77 plays a critical role in mediating the apoptotic effect of CCE9.

Transfected GFP-Nur77 was found exclusively in the nucleus of cells. However, GFP-Nur77 could be detected in both the nucleus and cytoplasm when cells were treated with CCE9 (Figure 2C). This observation suggested that CCE9 might be involved in inducing Nur77 cytoplasmic localization, the hallmark of the Nur77-Bcl-2 apoptotic pathway [7]. To confirm the effect of CCE9 on inducing the cytoplasmic localization of Nur77, we studied the subcellular localization of endogenous Nur77 in the presence or absence of CCE9 by immunostaining. In the absence of treatment, endogenous Nur77 protein was hardly detected. Consistent with its ability to induce Nur77 protein expression, CCE9 treatment resulted in a strong Nur77 staining both in the nucleus and cytoplasm of cells with predominant cytoplasmic staining in some cells (Figure 3A). To confirm the effect of CCE9 on inducing Nur77 cytoplasmic localization, cytosolic and nuclear fractions of cells treated with CCE9 were prepared and analyzed for levels of Nur77 protein by immunoblotting. In control cells, the level of Nur77 protein was low and it was exclusively detected in the nuclear fraction. When cells were treated with CCE9, the level of Nur77 protein was dramatically increased especially in the cytoplasmic fraction (Figure 3B). These results demonstrated that CCE9-induced Nur77 is predominantly cytoplasmic. To determine whether CCE9-induced Nur77 targeted at mitochondria, cells treated with CCE9 were stained with anti-Nur77 antibody and Mitotracker, a mitochondrialselective fluorescent label commonly used in confocal microscopy and flow cytometry [5]. Confocal microscopy showed that the distribution of Nur77 overlapped extensively with that of Mitotracker in cells treated with CCE9 (Figure 3C), suggesting that the cytoplasmic Nur77 induced by CCE9 could target mitochondria. Thus, CCE9 induces not only the expression of Nur77 but also its cytoplasmic localization and mitochondrial targeting.

Cytoplasmic Nur77 is known to bind Bcl-2 [7]. HeLa229 cells transfected with or without Bcl-2 siRNA were then used to determine whether cytoplasmic Nur77 associated with Bcl-2. Transfection of HeLa229 cells with Bcl-2 siRNA almost completely inhibited Bcl-2 expression in the absence or presence of CCE9 (Figure 3D). Immunostaining showed that CCE9 treatment resulted in an extensive colocalization of the cytoplasmic Nur77 with Bcl-2 in cells treated transfected with control siRNA (Figure 3E), suggesting their association in the cytoplasm. However, when cells were transfected with Bcl-2 siRNA,
CCE9-induced Nur77 protein was found predominantly in the nucleus (Figure 3E). These results demonstrated that CCE9-induced Nur77 protein associated with Bcl-2 in the cytoplasm, and importantly they suggested that Bcl-2 might act to retain CCE9-induced Nur77 in the cytoplasm likely through their interaction. The loop region of Bcl-2 is required for binding to Nur77 [7]. To determine whether Bcl-2 plays a role in the cellular distribution of Nur77 through their interaction, Bcl-2 mutants with different ability to bind Nur77 were transfected into Bcl-2-depleted HeLa cells to determine their effect of cytoplasmic localization of co-transfected Nur77. Immunostaining showed that transfection of full-length Bcl-2, Bcl-2/ $\Delta \mathrm{TM}$, and $\mathrm{Bcl}-2 / \Delta \mathrm{BH} 4$, which are known to interact with Nur77 [7], could retain co-transfected Nur77 in the cytoplasm.

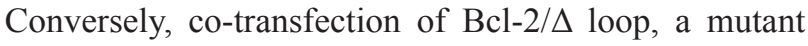
incapable of binding Nur77 [7], had no such an effect (Figure 3F). These results revealed not only the association of CCE9-induced cytoplasmic Nur77 with Bcl-2 but also a critical role of Bcl-2 expression in retaining Nur77 in the cytoplasm. We next determined whether the expression of Bcl-2 mediated the apoptotic effect of CCE9 using mouse embryonic fibroblasts (MEFs) and Bcl-2 knockout MEFs (Bcl-2--MEFs). Annexin V/PI staining showed that CCE9 treatment caused a significant early apoptosis in MEFs (Annexin $\mathrm{V}^{+} / \mathrm{PI} ; 16.51 \%$ ), while the same treatment hardly induced apoptosis in $\mathrm{Bcl}-2^{-/-} \mathrm{MEFs}\left(\right.$ Annexin $\mathrm{V}^{+} / \mathrm{PI}$ ; 3.73\%) (Figure 3G). Thus, the apoptotic effect of CCE9 also depends on Bcl-2 expression.

Nur77 interaction with Bcl-2 results in a Bcl-2 conformational change with its $\mathrm{BH} 3$ domain exposed [7]. Such a Bcl-2 conformation is pro-apoptotic and can be detected by antibody against the $\mathrm{BH} 3$ domain of $\mathrm{Bcl}-2$, anti-Bcl-2(BH3) [7, 15]. Despite the expression of $\mathrm{Bcl}-2$, HeLa229 cells were not immunostained by the anti-Bcl2(BH3) antibody, suggesting that Bcl-2 expressed in the cells was anti-apoptotic. In contrast, cells treated with CCE9 exhibited a strong Bcl-2(BH3) immunostaining (Figure $3 \mathrm{H}$ ), an indication of Bcl-2 conformational change from an anti-apoptotic to a pro-apoptotic molecule $[7,15]$. Pro-apoptotic Bcl-2 acts like a $\mathrm{BH} 3$-only protein known to activate $\operatorname{Bax}[8,15]$. Indeed, cells treated with CCE9 were strongly stained by the conformation-sensitive antiBax (6A7) antibody that recognizes an N-terminal epitope exposed after Bax activation [31] (Figure 3I), revealing the ability of CCE9 to activate Bax.

\section{CCE9 induction of apoptosis depends on its activation of $\mathrm{p38} \alpha$ MAPK}

A variety of protein kinases, including the MAPK family members, are known to regulate the Nur77Bcl-2 apoptotic pathway $[9,11,32,33]$. We previously reported that 3-Cl-AHPC activation of the Nur77-Bcl-2 apoptotic pathway involved its induction of CRM-1dependent Nur77 nuclear export through activation of 


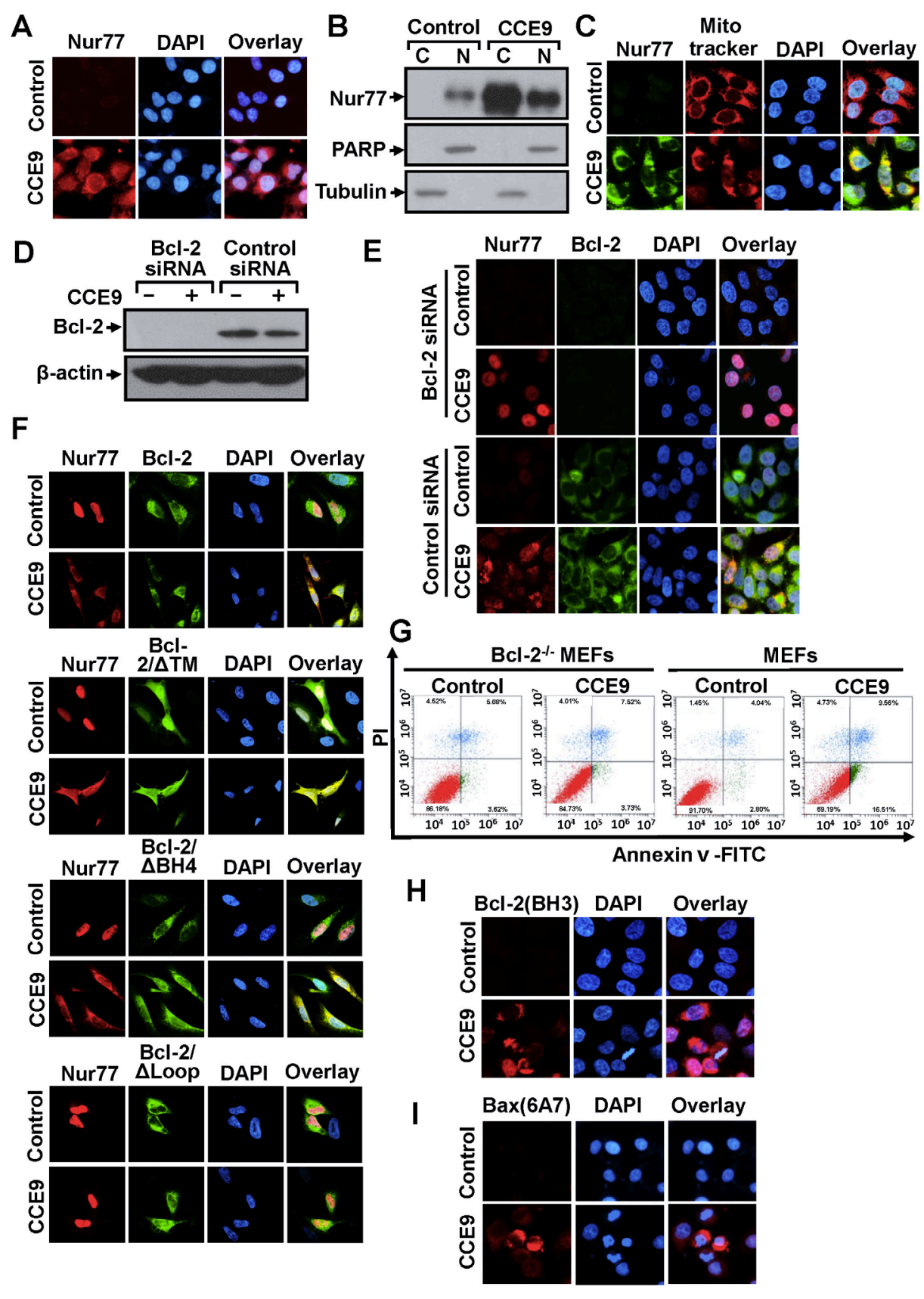

Figure 3: CCE9 induces Nur77 mitochondrial targeting, Bcl-2 colocalization, and Bcl-2 conformational change. (A) CCE9 induces cytoplasmic localization of Nur77. HeLa229 cells treated with $10 \mu \mathrm{M}$ CCE9 for 2 hr were subjected to immunostaining with anti-Nur77 antibody. Cells were co-stained with DAPI to visualize the nuclei. (B) Cellular fractionation. Nuclear and cytoplasmic fractions were prepared from HeLa229 cells treated with $10 \mu \mathrm{M}$ CCE9 for $2 \mathrm{hr}$, subjected to Western blotting analysis. The purity of cellular fraction was confirmed by using anti-PARP and anti-Tubulin antibodies. (C) Nur77 mitochondrial targeting. HeLa229 cells treated with 10 $\mu \mathrm{M}$ CCE9 for $2 \mathrm{hr}$, Nur77 expression was immunostained with anti-Nur77 antibody and analyzed by confocal microscopy. Cells were costained with Mitotraker to recognize the mitochondria and with DAPI to visualize the nuclei. (D-E) The effect of Bcl-2 siRNA transfection on Nur77 cytoplasmic localization. HeLa229 cells transfected with Bcl-2 siRNA or control siRNA for 48 hr and subsequently treated with CCE9 for $2 \mathrm{hr}$ were examined for Bcl-2 expression by Western blotting (D) or subcellular localization of Nur77 by immunostaining (E) Cells were co-stained with DAPI to visualize the nuclei. (F) Co-localization of Nur77 with Bcl-2 mutants. Bcl-2-depleted HeLa cells were transfected with Nur77 and the indicated Bcl-2 or mutants for $24 \mathrm{hr}$ and subsequently treated with CCE9 for $3 \mathrm{hr}$. Subcellular localization of Nur77 and Bcl-2 was examined by immunostaining. (G) The apoptotic effect of CCE9 is impaired in Bcl-2 knockout MEFs. MEFs or Bcl-2/- MEFs were treated with vehicle or $10 \mu \mathrm{M}$ CCE9 for $6 \mathrm{hr}$ and stained with Annexin V/PI. Apoptosis was analyzed by fluorescenceactivated cell sorting analysis. (H) Bcl-2 conformational change. HeLa229 cells treated with $10 \mu \mathrm{M}$ of CCE9 for $2 \mathrm{hr}$ were examined for Bcl-2 conformational change by anti-Bcl-2(BH3) antibody. Cells were also stained with DAPI to visualize the nucleus. (I) Bax activation. HeLa229 cells treated with $10 \mu \mathrm{M}$ of CCE9 for $2 \mathrm{hr}$ were examined for Bax activation by conformation-sensitive anti-Bax antibody, Bax (6A7). Cells were also stained with DAPI to visualize the nucleus. 
Jun N-terminal kinase (JNK) [9, 11, 32, 33]. Our current observation that inhibition of $\mathrm{Bcl}-2$ expression prevented CCE9 induction of Nur77 cytoplasmic localization (Figure 3E) suggested that CCE9 might regulate the Nur77-Bcl-2 apoptotic pathway through a different mechanism. Indeed, leptomycin B (LMB), an inhibitor of CRM-1-dependent nuclear export [34], had no effect on CCE9-induced Nur77 cytoplasmic localization (Figure 4A). LMB was also ineffective on the induction of PARP cleavage (Figure 4B) and apoptosis (Figure 4C) by CCE9. Furthermore, JNK activation known to promote Nur77 nuclear export [9, 11, $32,33]$ did not occur in HeLa229 cells treated with CCE9 (Figure 4D). Instead, we found that p38 $\alpha$ MAPK was rapidly and strongly activated by CCE9 in HeLa229 cells (Figure 4D). To determine the role of CCE9 activation of p38 $\alpha$ MAPK, we first used SB203580, a chemical inhibitor known to inhibit both $\mathrm{p} 38 \alpha$ and $\mathrm{p} 38 \beta$ MAPK. Treatment of HeLa229 cells with SB203580 but not a JNK inhibitor (SP600125) almost completely suppressed the effect of CCE9 on inducing PARP cleavage (Figure 4E). SB203580 also suppressed the effect of CCE9 on activating caspase-3 (Figure 4F) and apoptosis (Figure 4G). We next used p38 $\alpha$-specific siRNA approach to determine the effect of CCE9 on inducing p38 MAPK activation and apoptosis. Transfection of cells with p38 $\alpha$-specific siRNA completely inhibited the expression of p38 MAPK and its activation by CCE9 (Figure 4H). Thus, CCE9 mainly activates p $38 \alpha$ isoform but not other isoforms. Similar to the effect of SB203580, transfection of p38 $\alpha$-specific siRNA largely impaired the effect of CCE9 on inducing PARP (Figure $4 \mathrm{H})$. Thus, p38 $\alpha$ MAPK activation is essential for the apoptotic effect of CCE9.

\section{p38 $\alpha$ MAPK activation in Nur77 expression, Bcl-2 phosphorylation, and Bcl-2 conformational change}

Nur77 expression is required for the apoptotic effect of CCE9. The observation that p38 $\alpha$ MAPK inhibitor effectively suppressed CCE9-induced apoptosis suggested that p38 $\alpha$ MAPK might modulate the Nur7 apoptotic pathway. We first studied the effect of p38 $\alpha$ MAPK on CCE9 induction of Nur77 expression. Treatment of HeLa229 cells with the p38 $\alpha$ MAPK inhibitor SB203580 completely suppressed the dose-dependent induction of Nur77 expression (Figure 5A). Knocking down p38 $\alpha$ MAPK mRNA expression by transfecting p38 $\alpha$ MAPK siRNA also abolished the effect of CCE9 on inducing Nur77 expression and cytoplasmic localization (Figure 5B-5C). We also used p38 $\alpha$ knockout MEFs, p38 $\alpha^{-/-} \mathrm{MEFs,}$ to study the role of $\mathrm{p} 38 \alpha$ activation. Treatment of MEFs with CCE9 resulted in induction of Nur77 expression and PARP cleavage. In contrast, CCE9 had little effect on Nur77 expression and PARP cleavage in $\mathrm{p} 38 \alpha^{-/} \mathrm{MEFs}$ (Figure 5D). Thus, CCE9 induction of Nur77 expression requires its activation of $\mathrm{p} 38 \alpha$ MAPK. p38 $\alpha$ MAPK has been shown to phosphorylate Bcl-2 and regulate its survival activity [23-25]. To determine whether and how CCE9 activation of $\mathrm{p} 38 \alpha$ MAPK regulated the Nur77-Bcl-2 apoptotic pathway, we studied the possibility that $\mathrm{p} 38 \alpha$ MAPK regulated Nur77-dependent apoptosis by phosphorylating Bcl-2. Bcl-2 was immunoprecipitated with anti-Bcl-2 antibody from HeLa229 cells treated with or without CCE9, and the immunoprecipitated Bcl-2 was then analyzed for phosphorylation by anti-phosphor-Ser and antiphosphor-Thr antibodies. Our results showed that Bcl-2 immunoprecipitated from CCE9-treated cells reacted strongly with both anti-phospho-Ser and anti-phosphoThr antibodies, while Bcl-2 immunoprecipitates from nontreated cells did not. Moreover, Bcl-2 immunorecipitates from cells treated with CCE9 in the presence of SB203580 failed to react with anti-phospho-Ser and anti-phospho-Thr antibodies (Figure 5E). These results are consistent with previous report that $\mathrm{p} 38 \alpha$ MAPK could phosphorylate Bcl2 [23] and suggest that CCE9 is capable of inducing p38 $\alpha$ MAPK dependent phosphorylation of Bcl-2. Conversion of Bcl-2 from an anti-apoptotic to a pro-apoptotic molecule is characterized by the exposure of its $\mathrm{BH} 3$ domain [7]. Immunostaining showed that the apoptotic effect of CCE9 on inducing BH3 domain staining was strongly attenuated in cells treated with the p38 $\alpha$ MAPK inhibitor SB203580 (Figure 5F) or transfected with p38a MAPK siRNA (Figure 5G). Thus, p38 $\alpha$ MAPK activation by CCE9 plays a critical role in its induction of Bcl-2 conversion into a death factor.

\section{Regulation of Nur77-Bcl-2 interaction by p38a MAPK activation}

Bcl-2 phosphorylation by $\mathrm{p} 38 \alpha$ MAPK is mainly mediated by multiple phosphorylation sites in its loop region. As the loop of Bcl-2 is responsible for Bcl-2 interaction with Nur77 and subsequently Bcl2 conversion, we studied whether CCE9 activation of p38 $\alpha$ MAPK was involved in the regulation of Bcl-2 interaction with Nur77. Co-immunoprecipitation assays demonstrated that Nur77 induced by CCE9 treatment was co-immunoprecipitated together with phosphorylated Bcl-2 by anti-Bcl-2 antibody (Figure 6A), demonstrating their interaction. Such an interaction was not observed in cells transfected with p38 $\alpha$ MAPK siRNA (Figure 6A) or treated with SB203580 (Figure 6B), likely due to their inhibition of Nur77 expression and Bcl-2 phosphorylation. To exclude the effect of p38 $\alpha$ MAPK inhibition on Nur77 expression, we studied the interaction of transfected Nur77 and Bcl-2. Thus, Myc-tagged Bcl-2 and Flagtagged Nur77 were co-transfected into HeLa229 cells, and their interaction in the presence or absence of CCE9 was examined. Co-immunoprecipitation assays showed that transfected Flag-Nur77 and Myc-Bcl-2 strongly interacted when cells were treated with CCE9 in both 
A

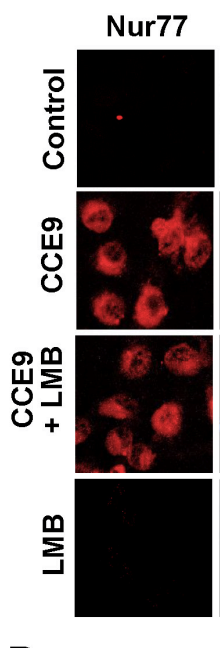

D

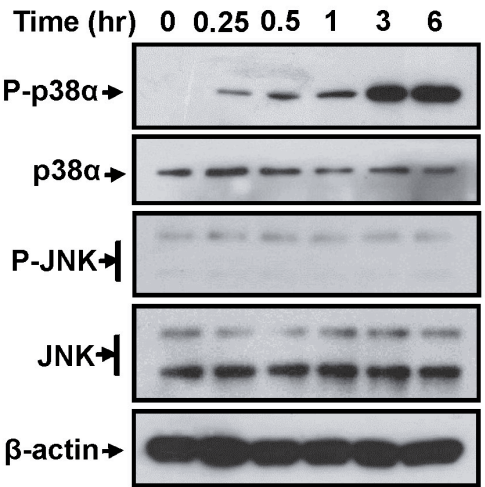

F

Cleaved caspase-3

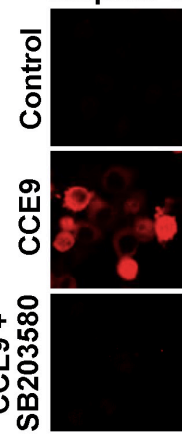

DAPI
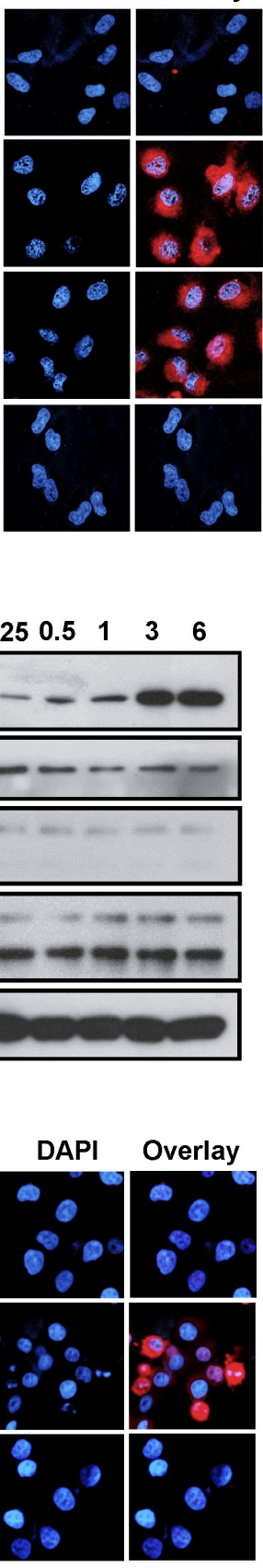
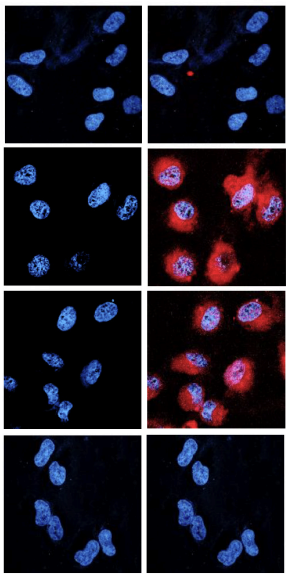

E

B

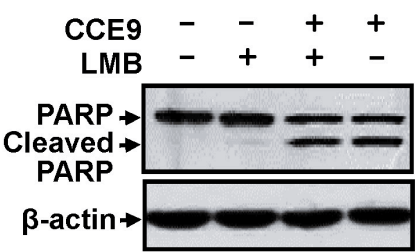

C
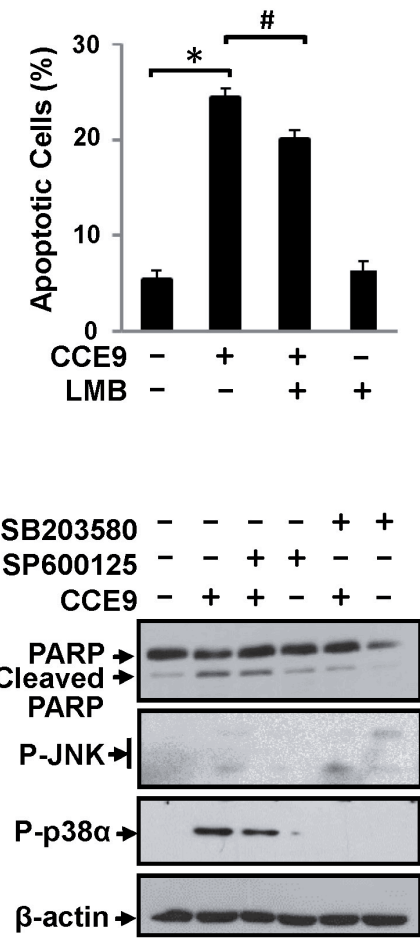

H

G
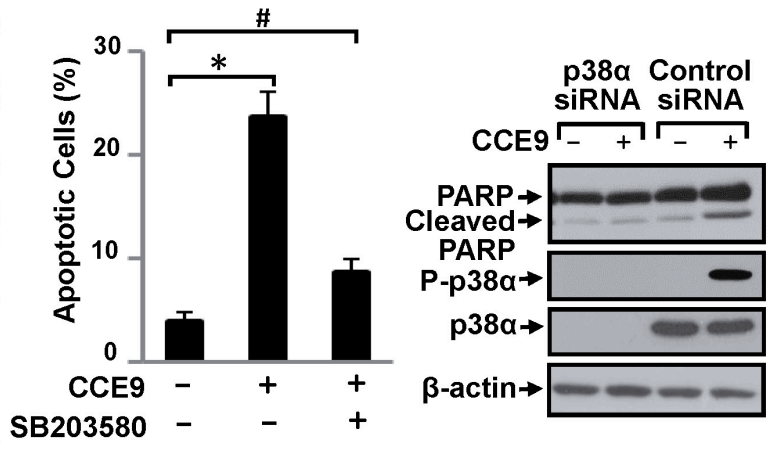

Figure 4: p38 $\alpha$ MAPK activation by CCE9 is required for apoptosis and Nur77 expression. (A) LMB fails to block CCE9induced Nur77 cytoplasmic localization. HeLa229 cells treated with $10 \mu \mathrm{M}$ of CCE9 in the presence or absence of LMB $(1 \mathrm{ng} / \mathrm{mL})$ for 2 hr were examined by immunostaining using anti-Nur77 antibody. (B-C) Effect of LMB on apoptosis induction by CCE9. HeLa229 cells treated with $10 \mu \mathrm{M}$ CCE9 in the presence or absence of LMB $(1 \mathrm{ng} / \mathrm{mL})$ for $3 \mathrm{hr}$ were studied for apoptosis by PARP cleavage by Western blotting (B) and DAPI staining, *, P $<0.01$ (vs. control); \#, P $>0.05$ (vs. CCE9 treatment) (C). (D) Induction of p38 MAPK activation by CCE9. HeLa229 cells treated with $10 \mu \mathrm{M}$ CCE9 for $0,0.25,0.5,1,3,6 \mathrm{hr}$ were analyzed for activation of $\mathrm{p} 38$ MAPK and JNK by Western blotting using antibody recognizing phosphorylated (P-) p38 MAPK or JNK. Total p38 MAPK and JNK was used as loading control. (E) Role of CCE9 activation of p38 MAPK in PAPR cleavage. HeLa229 cells treated for $3 \mathrm{hr}$ with $10 \mu \mathrm{M}$ CCE9 in the presence or absence of $25 \mu \mathrm{M}$ SB203580 or SP600125 were analyzed by Western blotting. (F-G) Effect of SB203580 on CCE9 apoptotic activities. HeLa229 cells treated for $3 \mathrm{hr}$ with $10 \mu \mathrm{M}$ CCE9 in the presence or absence of $25 \mu \mathrm{M} \mathrm{SB} 203580$ were examined by for caspase-3 activation by immunostaining with antibody recognizing the cleaved caspase-3 (F) and apoptosis by DAPI staining, *, P $<0.01$ (vs. control); \#, P $>0.05$ (vs. control) (G). (H) Effect of p38 $\alpha$ MAPK siRNA transfection on CCE9 apoptotic activities. HeLa229 cells transfected with p38 $\alpha$ MAPK siRNA or control siRNA for $48 \mathrm{hr}$, and treated with $10 \mu \mathrm{M}$ CCE9 for $3 \mathrm{hr}$ were analyzed by Western blotting were analyzed by Western blotting. 
A

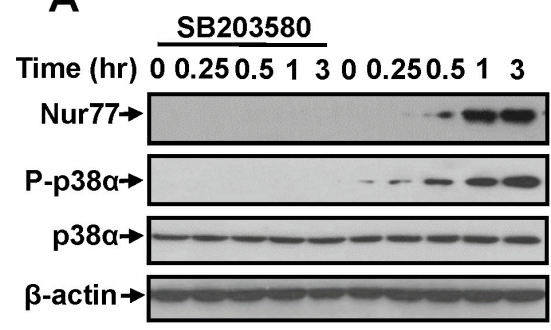

C

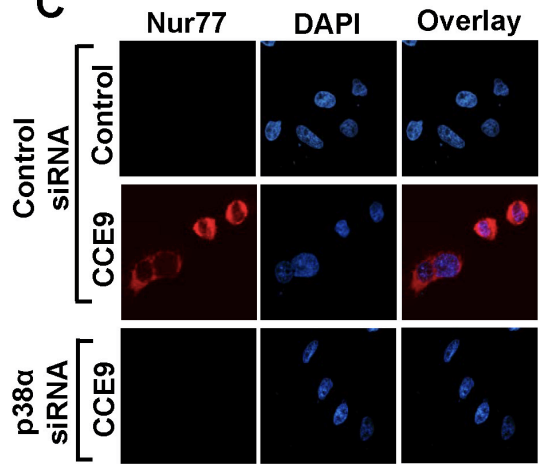

E

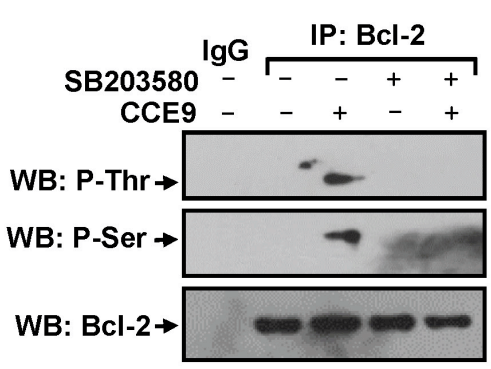

B

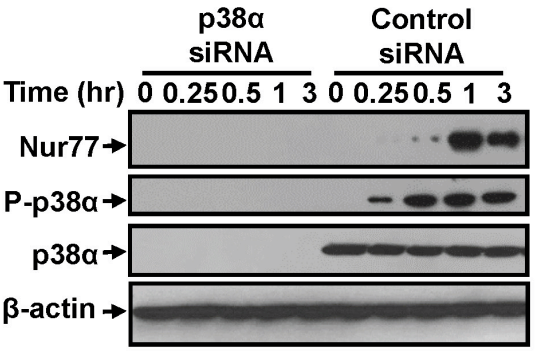

D

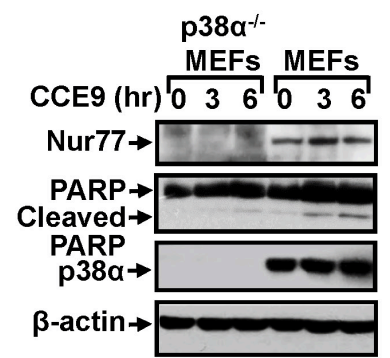

F

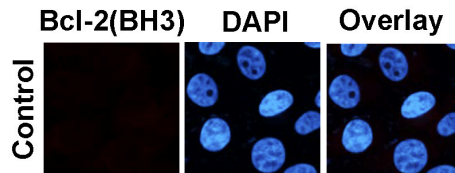

G
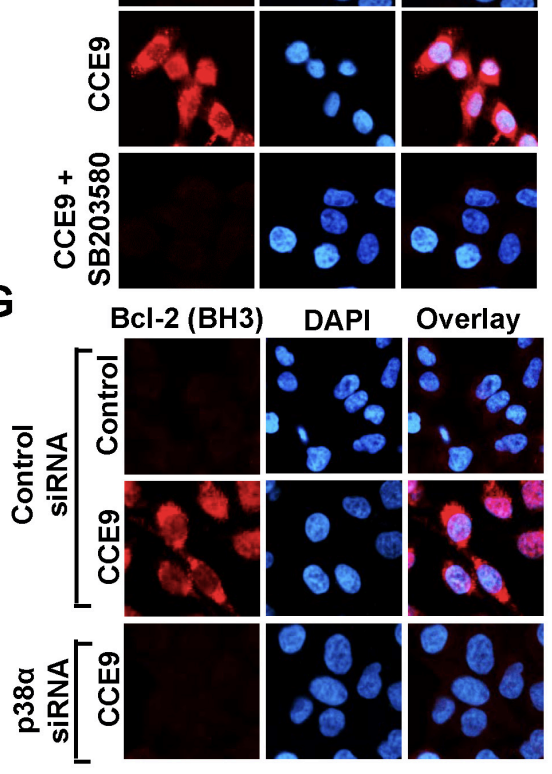

Figure 5: p38 $\alpha$ MAPK activation by CCE9 promotes Nur77 expression, Bcl-2 phosphorylation and Bcl-2 conformational change. (A) Effect of SB203580 on induction of Nur77 expression by CCE9. HeLa229 cells treated with $10 \mu \mathrm{M}$ CCE9 for 0, 0.25, 0.5, 1, $3 \mathrm{hr}$ in the presence or absence of $25 \mu \mathrm{M} \mathrm{SB} 203580$ were analyzed by Western blotting. (B-C) Effect of p38a MAPK siRNA transfection on induction of Nur77 expression by CCE9. HeLa229 cells transfected with p38 $\alpha$ MAPK siRNA or control siRNA for $48 \mathrm{hr}$, and treated with $10 \mu \mathrm{M}$ CCE9 for the indicated time were analyzed by Western blotting (B) and immunostaining (C) for Nur77 expression. (D) Effect of CCE9 on Nur77 expression and PARP cleavage in MEFs and p38 $\alpha$ MAPK $^{-}{ }^{-M E F s . ~ M E F s ~ o r ~ p 38 ~} \alpha$ MAPK ${ }^{-}$MEFs treated with $10 \mu \mathrm{M}$ CCE9 for $3 \mathrm{hr}$ and $6 \mathrm{hr}$ were analyzed by Western blotting for Nur77 protein expression and p38 MAPK activation. (E) CCE9 induced p38 $\alpha$ MAPK-dependent Bcl-2 phosphorylation. HeLa229 cells treated for $2 \mathrm{hr}$ with $10 \mu \mathrm{M}$ CCE9 in the presence or absence of $25 \mu \mathrm{M}$ SB203580 were subject to immunoprecipitated (IP) by using anti-Bcl-2 antibody. Immunoprecipitates were examined by Western blotting (WB) using anti-phosphorylated (P-) Ser and Thr antibodies. The same membranes were blotted with anti-Bcl-2 antibody to determine immunoprecipitation specificity. (F) Effect of SB203580 on CCE9 induced Bcl-2 conformational change. HeLa229 cells treated for 2 hr with $10 \mu \mathrm{M}$ CCE9 in the presence or absence of $25 \mu \mathrm{M} \mathrm{SB} 203580$, Bcl-2 conformational change by immunostaining with the antiBcl-2 (BH3) antibody. (G) Effect of p38 $\alpha$ MAPK siRNA transfection on CCE9 induced Bcl-2 conformational change. HeLa229 cells transfected with p38 $\alpha$ MAPK siRNA or control siRNA for $48 \mathrm{hr}$, and treated with $10 \mu \mathrm{M}$ CCE9 for $2 \mathrm{hr}$, Bcl-2 conformational change by immunostaining with the anti-Bcl-2 (BH3) antibody. 
A

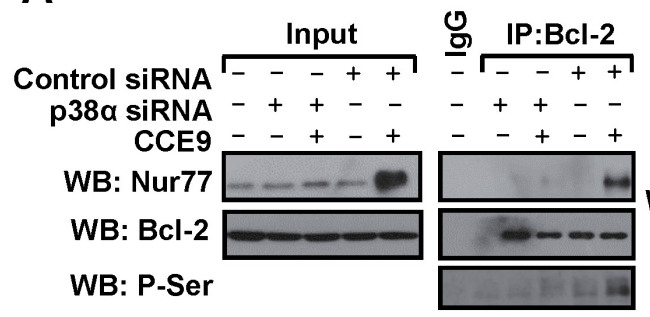

B

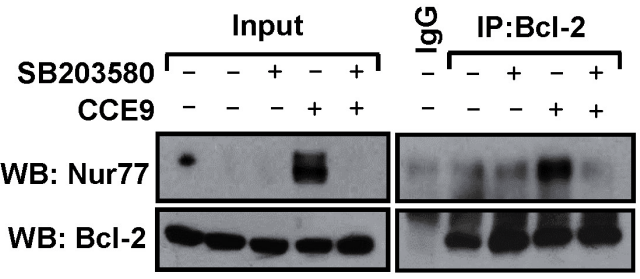

C

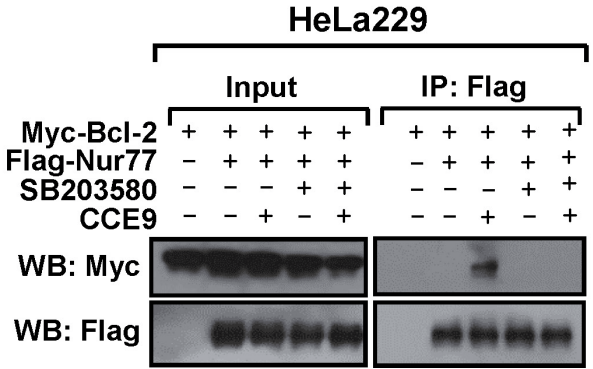

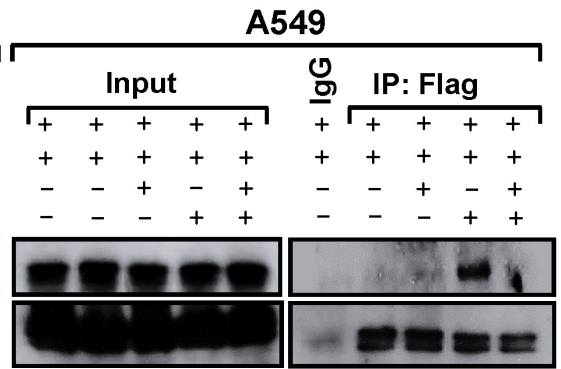

D

E
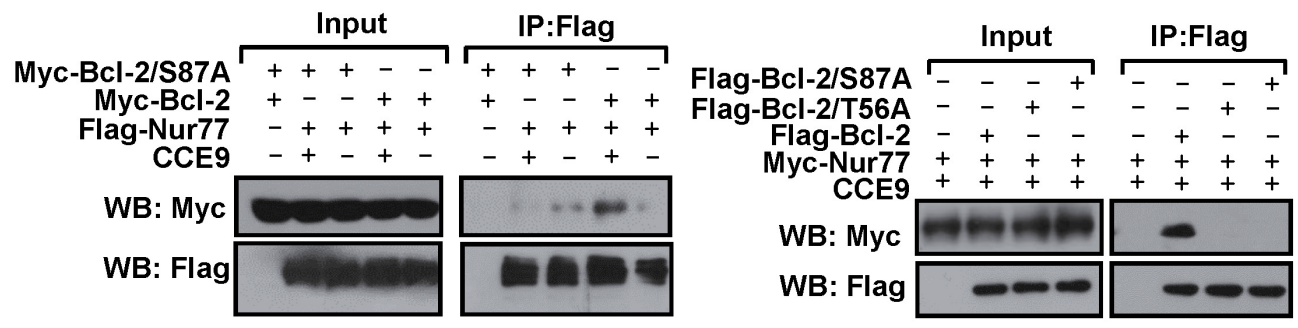

$\mathbf{F}$

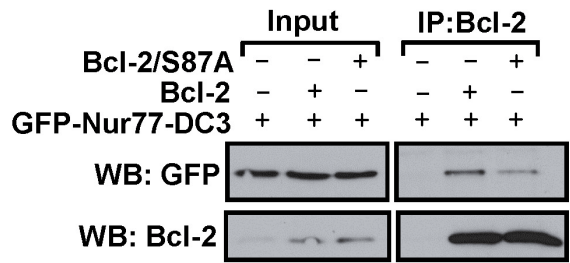

Figure 6: p38 $\alpha$ MAPK regulate Nur77-Bcl-2 interaction. (A) Transfection of p38 $\alpha$ MAPK siRNA inhibits the induction of Nur77 interaction with Bcl-2 by CCE9. HeLa229 cells were transfected with p38 $\mathrm{MAPK}$ siRNA for $48 \mathrm{hr}$, treated with $10 \mu \mathrm{M} \mathrm{CCE9}$ for $2 \mathrm{hr}$, and subjected to co-IP assays for Nur77 interaction with Bcl-2 by using anti-Bcl-2 antibody. Lysates and immunoprecipitates were examined by Western blotting using anti-Nur77, anti-Bcl-2, and anti-p-Ser antibodies. Input represents $10 \%$ of cell lysates used in the co-IP assays. (B) Effect of SB203580 on induction of Nur77 interaction with Bcl-2 by CCE9. HepG2 cells treated with $10 \mu \mathrm{M}$ CCE9 for $3 \mathrm{hr}$ in the presence or absence of $25 \mu \mathrm{M}$ SB203580 were immunoprecipitated by using anti-Bcl-2 antibody. Lysates and immunoprecipitates were examined by Western blotting using anti-Nur77 and anti-Bcl-2 antibodies. Input represents $10 \%$ of cell lysates used in the co-IP assays. (C) Inhibitory effect of SB203580 on CCE9-induced interaction of transfected Nur77 and Bcl-2. Myc-Bcl-2 (2 $\mu \mathrm{g})$ and Flag-Nur77 (2 $\mu \mathrm{g})$ expression vector alone or together were transfected into HeLa229 or A549 cells. Cell lysates were immunoprecipitated by using anti-Flag antibody. Lysates and immunoprecipitates were examined by Western blotting using anti-Myc and anti-Flag antibodies. Input represents $5 \%$ of cell lysates used in the co-IP assays. (D) Myc-Bcl-2 (2 $\mu \mathrm{g})$ or Myc-Bcl-2/S87A (2 $\mu \mathrm{g})$ and Flag-Nur77 $(2 \mu \mathrm{g})$ expression vector alone or together were transfected into HeLa229 cells. Cell lysates were immunoprecipitated by using anti-Flag antibody. Lysates and immunoprecipitates were examined by Western blotting using anti-Myc and anti-Flag antibodies. Input represents $5 \%$ of cell lysates used in the co-IP assays. (E) Flag-Nur77 (2 $\mu \mathrm{g})$ was transfected alone or together with Flag-Bcl-2 $(2 \mu \mathrm{g})$, Flag-Bcl-2/S87A (2 $\mu \mathrm{g})$, or Flag-Bcl-2/T56A (2 $\mu \mathrm{g})$ into Bcl2-depeleted HeLa cells. Cell lysates were immunoprecipitated by using anti-Flag antibody. Lysates and immunoprecipitates were examined by Western blotting using anti-Myc and anti-Flag antibodies. Input represents 5\% of cell lysates used in the co-IP assays. (F) Myc-Bcl-2 (2 $\mu \mathrm{g})$ or Myc-Bcl-2/S87A (2 $\mu \mathrm{g})$ and GFP-Nur77-DC3 $(2 \mu \mathrm{g})$ expression vector alone or together were transfected into HEK293T cells. Cell lysates were immunoprecipitated by using anti-Bcl-2 antibody. Lysates and immunoprecipitates were examined by Western blotting using anti-GFP and anti-Bcl-2 antibodies. Input represents $5 \%$ of cell lysates used in the co-IP assays. 
HeLa229 and A549 cells (Figure 6C). However, when cells were co-treated with SB203580, which did not affect the expression of transfected Flag-Nur77, CCE9-induced Nur77-Bcl-2 interaction was strongly inhibited (Figure 6C). Thus, CCE9-induced Nur77 interaction with Bcl2 likely involved its phosphorylation of Bcl-2 through its activation of $\mathrm{p} 38 \alpha$ MAPK. To further characterize the role of Bcl-2 phosphorylation in its interaction with Nur77, Ser87 and T56 in the loop of Bcl-2, which was shown to be phosphorylated by p38 $\alpha$ MAPK [23], were mutated into Ala. When Bcl-2/S87A, in which Ser87 was substituted with Ala, was analyzed for its interaction with Nur77 in HeLa229 cells, it failed to interact with Nur77 in cells treated with CCE9, while Bcl-2 strongly interacted with Nur77 under the same conditions (Figure 6D). CCE9 also showed much reduced effect on inducing Nur77 interaction with $\mathrm{Bcl}-2 / \mathrm{S} 87 \mathrm{~A}$ and another $\mathrm{Bc}-2$ mutant with Thr56 substituted with Ala (Bcl-2/T56A) in Bcl-2-depleted HeLa cells, as compared to its inducing effect on Nur77 interaction with wild-type Bcl-2 (Figure 6E). Interaction of Nur77-DC3, a Nur77 mutant that constitutively resides in the cytoplasm to interact with Bcl-2 [7, 15], with Bcl2/S87A was also reduced when compared to its binding to the wild-type Bcl-2 (Figure 6F). Together, these results demonstrate that $\mathrm{p} 38 \alpha$ MAPK phosphorylation of Bcl-2 at Ser87 and Thr56 plays an important role in its interaction with Nur77.

\section{DISCUSSION}

In studying the apoptotic effect of CCE9, we provided evidence here that it could induce apoptosis of various cancer cells through its activation of the Nur77Bcl-2 apoptotic pathway. This is illustrated by our data showing that the apoptosis induction by CCE9 was accompanied with its induction of Nur77 expression (Figure 1B-1E), Nur77 mitochondrial targeting (Figure 3C), and Nur77 interaction with Bcl-2 (Figure 3E).

The Nur77-Bcl-2 apoptotic pathway involves the translocation of Nur77 from the nucleus to the cytoplasm, which was shown to be mediated by a CRM-1-dependent nuclear export process [5, 33, 34]. Previous studies demonstrated that JNK activation was required for Nur77 nuclear export by certain apoptotic stimuli such as 3-ClAHPC [9, 11, 32, 33]. Our current study however showed that JNK was only moderately activated by CCE9 (Figure 4D). In addition, treatment of cells with LMB failed to block CCE9-induced Nur77 cytoplasmic accumulation (Figure 4A) and apoptosis induction (Figure 4B-4C). Furthermore, Bcl-2 expression was required for CCE9induced cytoplasmic accumulation of Nur77 protein (Figure 3D-3E). These observations together with our results that CCE9 could induce Nur77 interaction with cytoplasmic Bcl-2 (Figure 6) demonstrated that CCE9induced cytoplasmic localization of Nur77 is likely due to the cytoplasmic retention of the Nur77 protein by Bcl-
2 through protein-protein interaction. Our data that Bcl2 mutants capable of binding Nur77 could retain Nur77 in the cytoplasm while Bcl-2 mutant defective in Nur77 binding could not (Figure 3F) support this conclusion. Thus, CCE9 may employ a different mechanism to activate the Nur77-Bcl-2 pathway.

Indeed, we provide evidence that activation of $\mathrm{p} 38 \alpha$ MAPK by CCE9 plays a critical role in the regulation of the Nur77-Bcl-2 apoptotic pathway. The apoptotic effect of p38 $\alpha$ MAPK involving its modulation of death receptors, survival pathways, or pro- and anti-apoptotic Bcl-2 proteins has been well described [21, 22]. Diverse chemotherapeutic agents, including microtubuleperturbing drugs vinblastine, vincristine and paclitaxel and DNA damaging agent cisplatin, could stimulate apoptosis in a p38 $\alpha$ MAPK-dependent manner [21, 22]. Our results showed that CCE9 markedly activated p38 $\alpha$ MAPK in HeLa229 cells (Figure 4D), which coincided with its induction of Nur77 expression and apoptosis (Figure 4E, 5A-5B). Treatment of cells with the p38 $\alpha$ inhibitor (SB203580) or knocking down p38 $\alpha$ MAPK potently inhibited the ability of CCE9 to induce PARP cleavage (Figure 4E, 4H), caspase-3 activation (Figure $4 \mathrm{~F}$ ), and Bcl-2 conformational change (Figure 5F-5G) in HeLa229 Cells. Induction of PARP cleavage by CCE9 was also impaired in p38 $\alpha$ MAPK Knockout cells (Figure 5D). These data reveal a critical role of $\mathrm{p} 38 \alpha$ MAPK activation in mediating the apoptotic effects of CCE9.

The activation of the Nur77-Bcl-2 apoptotic pathway by CCE9 involves its induction of Nur77. Inhibition of p38 $\alpha$ MAPK activation by SB203580 (Figure 5A) or transfection of p38 $\alpha$ MAPK siRNA (Figure 5B) could inhibit the ability of CCE9 to induce Nur77 expression. Moreover, the level of Nur77 protein was dramatically reduced in $\mathrm{p} 38 \alpha^{-/} \mathrm{MEFs}$ as compared to the wild-type MEFs (Figure 5D). How p38 $\alpha$ MAPK acts to mediate the induction of Nur77 by CCE9 remains to be studied. It has been shown that $\mathrm{p} 38 \alpha$ MAPK activation by CD437, a Nur77 inducer, could phosphorylate MEF2 that serves as a transcriptional regulator of Nur77 [9]. Another study showed that induction of Nur77 by p38 $\alpha$ MAPK requires its activation of mitogen-and stress-activated protein kinase (MSK), which in turn phosphorylates CREB known to bind to two AP-1-like elements present in the Nur77 promoter [35]. However, our RT-PCR data (Figure 1F) demonstrated that CCE9 induction of Nur77 expression is unlikely mediated by its transcriptional regulation.

Our results revealed an important role of $\mathrm{p} 38 \alpha$ MAPK activation in promoting the interaction between Nur77 and Bcl-2. Cytoplasmic Nur77 is known to interact with Bcl-2, inducing a Bcl-2 conformational change that is pro-apoptotic [7]. CCE9-induced interaction of Nur77 with Bcl-2 was inhibited by transfection of $\mathrm{p} 38 \alpha$ MAPK siRNA (Figure 6A) or treatment with SB203580 (Figure 6B, 6C), demonstrating that CCE9 induction of the interaction of Nur77 with Bcl-2 depended on its activation of p38 $\alpha$ 
MAPK. Our results are in agreement with a previous report showing that inhibition of p $38 \alpha$ MAPK blocked the effect of $\alpha$-lipoic acid on inducing apoptosis and Nur77 cytoplasmic localization in vascular smooth muscle cells [11]. Binding of Bcl-2 to Nur77 is mediated by its natively unstructured loop region $[7,15]$. Phosphorylation in the loop region is known to suppress the anti-apoptotic function of Bcl-2 likely through its interference with Bcl-2 interaction with pro-apoptotic $\mathrm{Bcl}-2$ family proteins. Bcl-2 is phosphorylated by $\mathrm{p} 38 \alpha$ MAPK upon NGF withdrawal in memory B lymphocytes, leading to inhibition of its antiapoptotic activity and induction of cytochrome $c$ release [24]. Similarly, $\mathrm{H}_{2} \mathrm{O}_{2}$ induces p38 $\alpha$ MAPK-mediated Bcl-2 phosphorylation in adult rat cardiac myocytes [36], contributing to apoptosis [37]. Ser87 and Thr56 residues located in the unstructured loop region of $\mathrm{Bcl}-2$ have been shown to be phosphorylated by p38 $\alpha$ MAPK and the phosphorylation of these residues is associated with a decrease in the anti-apoptotic potential of $\mathrm{Bcl}-2$ protein [23]. Our findings that p38 $\alpha$ MAPK activation by CCE9 resulted in Bcl-2 phosphorylation (Figure 5E) and that mutating the p38 $\alpha$ phosphorylation sites in the loop of Bcl-2 reduced its interaction with Nur77 (Figure 6D-6F) reveal a critical role of $\mathrm{p} 38 \alpha$ MAPK phosphorylation of $\mathrm{Bcl}-2$ in the regulation of Bcl-2 interaction with Nur77, an event important for apoptosis induction. Thus, our results also provide new insight into the mechanism by which p38 $\alpha$ MAPK mediates the apoptotic effects of diverse chemotherapeutic agents.

\section{MATERIALS AND METHODS}

\section{Reagents}

Lipofectamine 2000 from Invitrogen (Carlsbad, CA, USA); goat anti-rabbit and anti-mouse secondary antibody conjugated to horseradish peroxidase from Thermo Fisher Scientific, Inc. (Waltham, MA, USA); anti-mouse/rabbit IgG conjugated with Cy3, anti-mouse/ rabbit IgG conjugated with FITC from Chemicon International (Temecula, CA, USA); anti-Nur77 (3960), anti-P-p38 (9215), anti-p38 (9212), anti-P-JNK (9251), anti-JNK (9252), anti-cleaved caspase-3 (9664), and antiTubulin (2144) antibodies from Cell Signal Technology; anti-Bcl-2 (sc-509), anti-Bax (6A7; sc-23959), and anti-Myc (9E10; sc-40) antibodies from Santa Cruz Biotechnology (Santa Cruz, CA, USA); anti-poly (ADPribose) polymerase antibody (PARP; 556494) from BD Biosciences (San Diego, CA, USA); anti- $\beta$-actin, antiFlag (M2), anti-P-Ser (p3430), and anti-P-Thr (p3555) antibodies from Sigma (St. Louis, MO, USA); SB203580, SP600125, leptomycin B (LMB), from Sigma; anti-Bcl-2 BH3 (AP1303a) from Abgent (San Diego, CA, USA); polyvinylidene difluoride membranes from Millipore (Billerica, MA, USA); and enhanced chemiluminescence reagents from GE Healthcare (Buckinghamshire, UK) and a cocktail of proteinase inhibitors from Roche (Meylan, France) were used in this study. All other chemicals used were commercial products of analytic grade obtained from Sigma (St. Louis, MO, USA).

\section{Cell culture and transient transfection}

HeLa cells, HeLa229 cells and HeLa Bcl-2 knockout cells were cultured in Eagle's Minimum Essential Medium containing 10\% fetal bovine serum (FBS), HepG2 cells, A549 cells, HEK293T cells, MEFs, p38 $\alpha$ MAPK $^{-/}$MEFs and Bcl-2/-MEFs were cultured in Dulbecco's modified Eagle's medium containing $10 \%$ fetal bovine serum (FBS). Subconfluent cells with exponential growth were used throughout the experiments. Transient transfection was performed using Lipofectamine 2000 (Invitrogen) according to the instructions of the manufacturer in regular growth medium.

\section{Plasmid constructions}

Expression vectors for Nur77, Bcl-2, Bcl-2/ $\triangle \mathrm{TM}$, $\mathrm{Bcl}-2 / \Delta \mathrm{BH} 4, \mathrm{Bcl}-2 / \Delta$ Loop and $\mathrm{Bcl}-2 / \mathrm{S} 87 \mathrm{~A}$ mutants were described previously $[7,15]$. Bcl-2/T56A mutant was generated by Fast Site-Directed Mutagenesis Kit from TIANGEN (Beijing, China).

\section{siRNA and transfections}

siRNAs against Nur77 (SASI_Hs02_00333289, SASI_Hs02_00333290, SASI_Hs02_00333291), p38 $\alpha$ MĀPK (SASI_Hs02_00331604, SASI_ Hs01_00018466, SASI_Hs02_00331605), and Bcl- $\overline{2}$ (SASI_Hs01_00119086, SASI_Hs01_001190867, SASI Hs01_001190868) and control siRNA used were from Sigma. $5 \mu \mathrm{L}$ aliquot of $20 \mu \mathrm{M}$ siRNA/well was transfected into cells in six-well plates using Lipofectamine 2000 (Invitrogen), according to the manufacturer's recommendations.

\section{Cell lysis and fractionation}

Cell lysates were prepared by lysing cells with modified radioimmunoprecipitation assay buffer containing $50 \mathrm{mM}$ Tris- $\mathrm{HCl}(\mathrm{pH} 7.4), 150 \mathrm{mM} \mathrm{NaCl}, 5$ mM EDTA, 1\% NP40, 0.5\% sodium deoxycholate, and $0.1 \%$ SDS with a cocktail of proteinase inhibitors on ice for $30 \mathrm{~min}$. For cellular fractionation, cells were lysed in cold buffer [10 mM HEPES-KOH (pH 7.9), $1.5 \mathrm{mM}$ $\mathrm{MgCl}_{2}, 10 \mathrm{mM} \mathrm{KCl}$, and $0.5 \mathrm{mM}$ DTT] with a cocktail of proteinase inhibitors on ice for $10 \mathrm{~min}$. The cytoplasmic fraction was collected by centrifuging at 6,000 rpm for 30 sec. Pellets containing nuclei were resuspended in cold high-salt buffer [20 mM HEPES-KOH (pH 7.9), $25 \%$ glycerol, $420 \mathrm{mM} \mathrm{NaCl}, 1.5 \mathrm{mM} \mathrm{MgCl}, 0.2 \mathrm{mM}$ EDTA, $0.5 \mathrm{mM}$ DTT], with a cocktail of proteinase 
inhibitors on ice for $30 \mathrm{~min}$. Cellular debris was removed by centrifugation at $12,000 \mathrm{rpm}$ at $4^{\circ} \mathrm{C}$ for $15 \mathrm{~min}$.

\section{Western blotting}

Equal amounts of the lysates were electrophoresed on an SDS-PAGE gel (8 or 12\%) and transferred onto polyvinylidene difluoride membranes, which were then blocked with 5\% nonfat milk in TBST [50 mM Tris-HCl (pH 7.4), $150 \mathrm{mM} \mathrm{NaCl}$, and $0.1 \%$ Tween 20] for $1 \mathrm{hr}$, incubated with various primary antibodies for $2 \mathrm{hr}$ at room temperature and incubated with secondary antibodies for $1 \mathrm{hr}$. Immunoreactive products were detected by chemiluminescence with an enhanced chemiluminescence system (GE Healthcare).

\section{Co-immunoprecipitation assays}

For co-immunoprecipitation (co-IP) assay [7, 15, 38], cell lysates were incubated with the appropriate antibody for $1 \mathrm{hr}$, and subsequently incubated with protein A-Sepharose beads for $1 \mathrm{hr}$. The protein-antibody complexes recovered on beads were subjected to western blotting using appropriate antibodies after separation by SDS-polyacrylamide gel electrophoresis.

\section{Immunofluorescence microscopy}

Cells mounted on glass slides were permeabilized with PBS containing $0.1 \%$ Triton X-100 for $15 \mathrm{~min}$, and blocked with $1 \%$ bovine serum albumin (BSA) in PBS for $30 \mathrm{~min}$ at room temperature, followed with incubation with various primary antibodies at $37^{\circ} \mathrm{C}$ for $1 \mathrm{hr}$ and detected by FITC-labeled anti-mouse/rabbit IgG (Chemicon International) or anti-mouse/rabbit IgG conjugated with $\mathrm{Cy} 3$ (Chemicon International) at room temperature for 30 min. Cells were co-stained with 4'6'-diamidino-2phenylindole (DAPI) (Sigma) to visualize nuclei. The images were taken under an LSM-510 confocal laser scanning microscope system (Carl Zeiss, Oberkochen, Germany).

\section{Apoptosis assays}

For DAPI staining, cells were collected and incubated in PBS containing $50 \mu \mathrm{g} / \mathrm{mL}$ of DAPI and $100 \mu \mathrm{g} / \mathrm{mL}$ of DNase-free RNase A at $37^{\circ} \mathrm{C}$ for $20 \mathrm{~min}$. Apoptotic cells were identified with typical morphology of shrinkage of the cytoplasm, membrane blebbing, and nuclear condensation and/or fragmentation. At least 300 cells from more than five random microscopic fields were counted by two independent investigators.

\section{Reverse transcription-PCR analysis}

Total RNAs were isolated by Trizol LS. The firststrand synthesis was performed with Revert Aid First-
Strand cDNA synthesis kits (Fermentas) according to the instructions of the manufacturer. The primers include those for Nur77 (forward primer, 5'-TCA TGG ACG GCT ACA CAG-3', reverse primer, 5'-GTA GGC ATG GAA TAG CTC-3') and $\beta$-actin (forward primer, 5'-CTG GAG AAG AGC TAC GAG-3', reverse primer, 5'-TGA TGG AGT TGA AGG TAG-3'). PCR reactions were performed in Eppendorf AG 22331 Hamburg (Eppendorf), and analyzed by electrophoresis using $2 \%$ agarose gels. Gel images were captured with a Gel logic 200 system (Kodak).

\section{Flow cytometric analysis}

Apoptosis was determined by dual staining using Annexin V/FITC and propidium iodide (Invitrogen). Briefly, cells were seeded into 24-well cell culture plates $\left(1 \times 10^{5}\right.$ cells/well $)$ and treated with CCE9 for $6 \mathrm{hr}$. Cells were dissociated from wells with $0.25 \%$ trypsin, spun at $1,500 \mathrm{rpm}$ for $5 \mathrm{~min}$, resuspended in Annexin $\mathrm{V}$ binding buffer, and stained with $1 \mu \mathrm{L}$ Annexin V/FITC for $15 \mathrm{~min}$ and $1 \mu \mathrm{L}$ propidium iodide for $1 \mathrm{~min}$. Cells were analyzed using the FACSCalibur System (BD Biosciences, San Jose, CA, USA). The relative proportion of Annexin V-positive cells, representing apoptotic cells, was determined using FlowJo software (FlowJo LLC, Ashland, OR, USA).

\section{Statistical analysis}

Data were expressed as mean $\pm \mathrm{SD}$. Each assay was repeated in triplicate in three independent experiments. Statistical significance of differences between groups was analyzed by using Student's t test or ANOVA analysis. $\mathrm{p}<0.05$ was considered significant.

\section{Abbreviations}

MAPK, mitogen-activated protein kinase; PARP, poly ADP-ribose polymerase; siRNA, small interfering RNA; BH domain, Bcl-2 homology domain; JNK, c-Jun N-terminal kinase; DAPI, 4'6'-diamidino-2-phenylindole; $\mathrm{LMB}$, leptomycin $\mathrm{B} ; \mathrm{H}_{2} \mathrm{O}_{2}$, hydrogen peroxide; GFP, green fluorescent protein.

\section{Author contributions}

X.-K.Z., X.-S.Y. and J.L. researched idea and study design. J.L., G.-H.W., Y.-H.D., Y.D., Y.B., M.H. and Y.-Q.Z. performed data collection. X.-K.Z., J.L., G.H.W., F.J., M.L. and H.Z. analyzed and interpreted data. All authors read and agreed to the final version of the manuscript.

\section{ACKNOWLEDGMENTS}

We thank Dr. Jiahuai Han for kindly providing the p38 $\alpha$ MAPK $^{-/}$MEF cells. 


\section{CONFLICTS OF INTEREST}

The Authors do not have any conflicts of interest.

\section{FUNDING}

This work was supported by the National Natural Science Foundation of China (NSFC-81502406, NSFC91429306, NSFC-U1405229, NSFC-81672749, NSFC81673320, NSFC-91129302, NSFC-81370097, NSFC81301705, NSFC-31271453 and NSFC-81670709), the Natural Science Foundation of Fujian Province of China (2015J05169, 2017YZ0002, 2015J01065), Fujian young teacher education research project (JA14009), and Regional Demonstration of Marine Economy Innovative Development Project (14PYY051SF04 and 16PYY007SF17).

\section{REFERENCES}

1. Moll UM, Marchenko N, Zhang XK. p53 and Nur77/TR3 - transcription factors that directly target mitochondria for cell death induction. Oncogene. 2006; 25:4725-4743.

2. Zhang XK. Targeting Nur77 translocation. Expert Opin Ther Targets. 2007; 11:69-79.

3. Lee SO, Li X, Khan S, Safe S. Targeting NR4A1 (TR3) in cancer cells and tumors. Expert Opin Ther Targets. 2011; 15:195-206

4. Mohan HM, Aherne CM, Rogers AC, Baird AW, Winter DC, Murphy EP. Molecular pathways: the role of NR4A orphan nuclear receptors in cancer. Clin Cancer Res. 2012; 18:3223-3228.

5. Li H, Kolluri SK, Gu J, Dawson MI, Cao X, Hobbs PD, Lin B, Chen G, Lu J, Lin F, Xie Z, Fontana JA, Reed JC, Zhang X. Cytochrome c release and apoptosis induced by mitochondrial targeting of nuclear orphan receptor TR3. Science. 2000; 289:1159-1164.

6. Dawson MI, Hobbs PD, Peterson VJ, Leid M, Lange CW, Feng KC, Chen G, Gu J, Li H, Kolluri SK, Zhang X, Zhang Y, Fontana JA. Apoptosis induction in cancer cells by a novel analogue of 6-[3-(1- adamantyl)-4-hydroxyphenyl]2-naphthalenecarboxylic acid lacking retinoid receptor transcriptional activation activity. Cancer Res. 2001; 61:4723-4730.

7. Lin B, Kolluri SK, Lin F, Liu W, Han YH, Cao X, Dawson MI, Reed JC, Zhang XK. Conversion of Bcl-2 from protector to killer by interaction with nuclear orphan receptor Nur77/TR3. Cell. 2004; 116:527-540.

8. Liu J, Zhou W, Li SS, Sun Z, Lin B, Lang YY, He JY, Cao X, Yan T, Wang L, Lu J, Han YH, Cao Y, et al. Modulation of orphan nuclear receptor Nur77-mediated apoptotic pathway by acetylshikonin and analogues. Cancer Res. 2008; 68:8871-8880.
9. Holmes WF, Soprano DR, Soprano KJ. Early events in the induction of apoptosis in ovarian carcinoma cells by CD437: activation of the p38 MAP kinase signal pathway. Oncogene. 2003; 22:6377-6386.

10. Yang H, Nie Y, Li Y, Wan YJ. ERK1/2 deactivation enhances cytoplasmic Nur77 expression level and improves the apoptotic effect of fenretinide in human liver cancer cells. Biochem Pharmacol. 2011; 81:910-916.

11. Kim HJ, Kim JY, Lee SJ, Kim HJ, Oh CJ, Choi YK, Lee HJ, Do JY, Kim SY, Kwon TK, Choi HS, Lee MO, Park IS, et al. alpha-Lipoic acid prevents neointimal hyperplasia via induction of p38 mitogen-activated protein kinase/Nur77mediated apoptosis of vascular smooth muscle cells and accelerates postinjury reendothelialization. Arterioscler Thromb Vasc Biol. 2010; 30:2164-2172.

12. Wang A, Rud J, Olson CM Jr, Anguita J, Osborne BA. Phosphorylation of Nur77 by the MEK-ERK-RSK cascade induces mitochondrial translocation and apoptosis in $\mathrm{T}$ cells. J Immunol. 2009; 183:3268-3277.

13. Thompson J, Winoto A. During negative selection, Nur77 family proteins translocate to mitochondria where they associate with Bcl-2 and expose its proapoptotic $\mathrm{BH} 3$ domain. J Exp Med. 2008; 205:1029-1036.

14. Maddika S, Booy EP, Johar D, Gibson SB, Ghavami S, Los M. Cancer-specific toxicity of apoptin is independent of death receptors but involves the loss of mitochondrial membrane potential and the release of mitochondrial celldeath mediators by a Nur77-dependent pathway. J Cell Sci. $2005 ; 118: 4485-4493$.

15. Kolluri SK, Zhu X, Zhou X, Lin B, Chen Y, Sun K, Tian X, Town J, Cao X, Lin F, Zhai D, Kitada S, Luciano F, et al. A short Nur77-derived peptide converts Bcl-2 from a protector to a killer. Cancer Cell. 2008; 14:285-298.

16. Thompson J, Burger ML, Whang H, Winoto A. Protein kinase $\mathrm{C}$ regulates mitochondrial targeting of Nur77 and its family member Nor-1 in thymocytes undergoing apoptosis. Eur J Immunol. 2010; 40:2041-2049.

17. Liu S, Yu H, Kumar SM, Martin JS, Bing Z, Sheng W, Bosenberg M, Xu X. Norcantharidin induces melanoma cell apoptosis through activation of TR3 dependent pathway. Cancer Biol Ther. 2011; 12:1005-1014.

18. Ferlini C, Cicchillitti L, Raspaglio G, Bartollino S, Cimitan S, Bertucci C, Mozzetti S, Gallo D, Persico M, Fattorusso C, Campiani G, Scambia G. Paclitaxel directly binds to Bcl-2 and functionally mimics activity of Nur77. Cancer Res. 2009; 69:6906-6914.

19. Blagosklonny MV. Unwinding the loop of Bcl-2 phosphorylation. Leukemia. 2001; 15:869-874.

20. Haldar S, Jena N, Croce CM. Inactivation of Bcl-2 by phosphorylation. Proc Natl Acad Sci U S A. 1995; 92:4507-4511.

21. Ono K, Han J. The p38 signal transduction pathway: activation and function. Cellular signalling. 2000; 12:1-13. 
22. Hui L, Bakiri L, Stepniak E, Wagner EF. p38alpha: a suppressor of cell proliferation and tumorigenesis. Cell Cycle. 2007; 6:2429-2433.

23. De Chiara G, Marcocci ME, Torcia M, Lucibello M, Rosini P, Bonini P, Higashimoto Y, Damonte G, Armirotti A, Amodei S, Palamara AT, Russo T, Garaci E, Cozzolino F. Bcl-2 Phosphorylation by p38 MAPK: identification of target sites and biologic consequences. J Biol Chem. 2006; 281:21353-21361.

24. Torcia M, De Chiara G, Nencioni L, Ammendola S, Labardi D, Lucibello M, Rosini P, Marlier LN, Bonini P, Dello Sbarba P, Palamara AT, Zambrano N, Russo T, et al. Nerve growth factor inhibits apoptosis in memory B lymphocytes via inactivation of $\mathrm{p} 38 \mathrm{MAPK}$, prevention of $\mathrm{Bcl}-2$ phosphorylation, and cytochrome c release. J Biol Chem. 2001; 276:39027-39036.

25. Ishikawa Y, Kusaka E, Enokido Y, Ikeuchi T, Hatanaka H. Regulation of Bax translocation through phosphorylation at Ser-70 of Bcl-2 by MAP kinase in NO-induced neuronal apoptosis. Mol Cell Neurosci. 2003; 24:451-459.

26. Na Y. Recent cancer drug development with xanthone structures. J Pharm Pharmacol. 2009; 61:707-712.

27. Pinto MM, Sousa ME, Nascimento MS. Xanthone derivatives: new insights in biological activities. Curr Med Chem. 2005; 12:2517-2538.

28. Zhou S, Kestell P, Baguley BC, Paxton JW. 5,6-dimethylxanthenone-4-acetic acid (DMXAA): a new biological response modifier for cancer therapy. Investigational new drugs. 2002; 20:281-295.

29. Akao Y, Nakagawa Y, Iinuma M, Nozawa Y. Anti-cancer effects of xanthones from pericarps of mangosteen. Int J Mol Sci. 2008; 9:355-370.

30. Duan YH, Dai Y, Wang GH, Zhang X, Chen HF, Chen JB, Yao XS, Zhang XK. Bioactive xanthones from the stems of
Cratoxylum formosum ssp. pruniflorum. J Nat Prod. 2010; 73:1283-1287.

31. Nechushtan A, Smith CL, Hsu YT, Youle RJ. Conformation of the Bax C-terminus regulates subcellular location and cell death. EMBO J. 1999; 18:2330-2341.

32. Han YH, Cao X, Lin B, Lin F, Kolluri SK, Stebbins J, Reed JC, Dawson MI, Zhang XK. Regulation of Nur77 nuclear export by c-Jun N-terminal kinase and Akt. Oncogene. 2006; 25:2974-2986.

33. Katagiri Y, Takeda K, Yu ZX, Ferrans VJ, Ozato K, Guroff G. Modulation of retinoid signalling through NGF-induced nuclear export of NGFI-B. Nat Cell Biol. 2000; 2:435-440.

34. Cao X, Liu W, Lin F, Li H, Kolluri SK, Lin B, Han YH, Dawson MI, Zhang XK. Retinoid X receptor regulates Nur77/TR3-dependent apoptosis [corrected] by modulating its nuclear export and mitochondrial targeting. Mol Cell Biol. 2004; 24:9705-9725.

35. Darragh J, Soloaga A, Beardmore VA, Wingate AD, Wiggin GR, Peggie M, Arthur JS. MSKs are required for the transcription of the nuclear orphan receptors Nur77, Nurr1 and Nor1 downstream of MAPK signalling. Biochem J. 2005; 390:749-759.

36. Cheng Z, Volkers M, Din S, Avitabile D, Khan M, Gude N, Mohsin S, Bo T, Truffa S, Alvarez R, Mason M, Fischer $\mathrm{KM}$, Konstandin $\mathrm{MH}$, et al. Mitochondrial translocation of Nur77 mediates cardiomyocyte apoptosis. Eur Heart J. 2011; 32:2179-2188.

37. Markou T, Dowling AA, Kelly T, Lazou A. Regulation of Bcl-2 phosphorylation in response to oxidative stress in cardiac myocytes. Free Radic Res. 2009; 43:809-816.

38. Zhou H, Liu W, Su Y, Wei Z, Liu J, Kolluri SK, Wu H, Cao Y, Chen J, Wu Y, Yan T, Cao X, Gao W, et al. NSAID sulindac and its analog bind RXRalpha and inhibit RXRalpha-dependent AKT signaling. Cancer Cell. 2010; 17:560-573. 